\title{
Zoometria como ferramenta de caracterização morfoestrutural genética em fêmeas da raça Sindi no Brasil
}

Mariz, T.M. de A. ${ }^{\circledR}$; Gonzaga Neto, S. ${ }^{2}$; Pimenta Filho, E.P. ${ }^{2}$; Ribeiro, M.N.. ${ }^{3}$; Givisiez, P.E.N. ${ }^{2}$; Cândido, E.P. ${ }^{2}$; Bezerra, L.R. ${ }^{4}$ e de Lima Júnior, D.M.'

'Universidade Federal de Alagoas. Campus Arapiraca. Curso de Zootecnia. Arapiraca. AL. Brasil.

${ }^{2}$ Universidade Federal da Paraíba. Campus II. Departamento de Zootecnia. Areia. PB. Brasil.

${ }^{3}$ Universidade Federal Rural de Pernambuco. Departamento de Zootecnia. Recife. PE. Brasil.

${ }^{4}$ Universidade Federal do Piauí. CPCE. Departamento de Ciência Animal. Bom Jesus. PI. Brasil.

PALAVRAS CHAVE ADICIONAIS

Classificação linear.

Linhagem.

Zebuínos.

\section{RESUMO}

Este trabalho foi executado com a finalidade de acrescentar elementos objetivos na avaliação das diferenças genéticas entre as duas bases conhecidas da raça Sindi no Brasil. Para isso, realizou-se a caracterização zoométrica de vacas Sindi utilizando medidas cranianas, morfoestruturais e índices corporais de fêmeas adultas de cinco rebanhos formados a partir das duas bases. Os dados foram submetidos a uma análise de variância e teste de médias (Tukey, $5 \%$ de probabilidade) e posteriormente a uma análise multivariada de componentes principais. Verificou-se diferença estatística significante entre as médias obtidas para a maioria das características estudadas, principalmente nas medidas morfoestruturais e nos índices corporais, demonstrando claramente a formação de grupos distintos em relação à base de formação genética dos rebanhos, o que sugere a presença de duas linhas genéticas distintas da raça no Brasil.

\section{Morphometry as a morphostructural characterization tool in Red Sindhi cattle females in Brazil}

\section{SUMMARY}

\author{
ADDITIONAL KEYWORDS \\ Linear classification. \\ Lineage. \\ Zebu cattle.
}

\section{INFORMACIÓN}

\section{Cronología del artículo.}

Recibido/Received: 02.07.2015

Aceptado/Accepted: 21.01 .2016

On-line: 16.03 .2016

Correspondencia a los autores/Contact e-mail:

tobyasmariz@hotmail.com
This study aims at pointing out the existing genetic differences between the Red Sindhi breed two basic groups in Brazil. This was made through the zoometric characterization of Red Sindhi cows by means of using their cranium and morphostructural measurements and body index of adult females from five herds proceeding from both distinct origins. Data were subjected to an analysis of variance and a means test (5\% probability level by Tukey's test) and were subjected to a multivariate analysis. There were significant differences between the majority of the studied characteristics, especially morphostructural measurements and body index. The results have clearly evidenced the presence of different groups compared to the genetic basis, suggesting the presence of two distinct genetic lineages of this breed in Brazil.

\section{INTRODUÇÃO}

A introdução da raça Sindi no Brasil ocorreu, num primeiro momento, em importações comerciais feitas nas décadas de 1920 e 1930, além de um segundo momento, de uma importação oficial feita pelo Instituto Agronômico do Norte (IAN) no ano de 1952.

Os animais do IAN se espalharam ao longo dos anos em diversas instituições oficiais, sendo seus descendentes diretos atualmente criados/mantidos na Empresa Estadual de Pesquisa Agropecuária da Paraíba (EMEPA-PB) e na Embrapa Semiárido (CPTSA). O criador José Cezário Castilho formou o que se tornaria, por muitos anos, o maior rebanho da raça no Brasil, a partir de animais provenientes das importações ditas comerciais e de indivíduos oriundos da importação feita pelo IAN, com seleção perpetuada até hoje por seus familiares (Leite, 2001).

Atualmente, a grande maioria do rebanho nacional encontra-se na região Nordeste, podendo-se afirmar que os mais de 6000 animais existentes têm como base de formação esses criatórios precursores, já que nenhuma importação de material genético desse grupamento racial para o país ocorreu desde aquelas anteriormente descritas. 
Tabela I. Resumo descritivo dos rebanhos analisados quanto a sua origem de formação $(\mathrm{N}=$ número de fêmeas avaliadas) (Descriptive summary of the herds analysed according to their formation origin ( $\mathrm{N}=$ number of studied females)).

\begin{tabular}{|c|c|c|c|c|}
\hline Rebanho & Local & Origem & Código & $\mathrm{N}$ \\
\hline EMEPA-PB & Alagoinha-PB & Importação 1952 & 1 & 42 \\
\hline $\begin{array}{l}\text { CPATSA/ } \\
\text { EMBRAPA }\end{array}$ & Petrolina-PB & Importação 1952 & 2 & 50 \\
\hline $\begin{array}{l}\text { Faz. Lagoa } \\
\text { Formosa }\end{array}$ & $\begin{array}{l}\text { Novo Horizonte- } \\
\text { SP }\end{array}$ & Família Castilho & 3 & 40 \\
\hline Nupeárido/UFCG & Patos-PB & Família Castilho & 4 & 40 \\
\hline Faz. Fazendinha & Mogeiro-PB & Mista & 5 & 49 \\
\hline
\end{tabular}

A raça passou por problemas de registro junto à $\mathrm{ABCZ}$ por alguns anos, mas com a retomada dos trabalhos pela associação de criadores, houve um crescimento do número de registros genealógicos a partir do ano de 2004. Contudo, poucos estudos com vista a acompanhar o crescimento da raça no país foram feitos. Partindo-se do princípio de que populações geneticamente distintas expressam essa dessemelhança por meio de medidas e índices corporais (Torres e Jardim, 1982; Bodó, 1990; Herrera, 2002; Ribeiro, 2004), admitese a caracterização zoométrica como uma ferramenta eficaz para identificação dessa diferença.

Objetivou-se, com este trabalho, acrescentar subsídios para a indicação de diferença genética entre as duas bases de formação da raça Sindi no Brasil.

\section{MATERIAL E MÉTODOS}

O estudo foi realizado utilizando fêmeas adultas de cinco rebanhos da raça Sindi, a fim de estabelecer parâmetros que vislumbrassem características zoométricas comparativamente representativas para o rebanho nacional.

Para tanto, foram avaliados os rebanhos oficiais da Empresa Estadual de Pesquisa Agropecuária da Paraíba (EMEPA-PB), no município de Alagoinha, Paraíba, e da Embrapa Semiárido (CPATSA), no município de Petrolina, Pernambuco, como rebanhos de base de formação histórica com descendência direta dos animais provenientes da importação oficial feita pelo Instituto Agronômico do Norte (IAN), em 1952. Por outro lado, o rebanho da Fazenda Lagoa Formosa, pertencente ao Sr. Adaldio Castilho e localizado no município de Novo Horizonte-SP, e o rebanho da Fazenda Nupeárido, pertencente ao Centro de Saúde e Tecnologia Rural da Universidade Federal de Campina Grande (Patos-PB), foram tomados como representação direta da seleção iniciada por José Cezário Castilho, que se espalhou pelo país através de parcerias firmadas no passado. Por fim, foi avaliado o rebanho do Dr. Mário Silveira, da fazenda Fazendinha (Mogeiro-PB), como forma de contemplar um rebanho comercial mais recentemente formado, oriundo de animais fundadores dessas duas bases genéticas presentes no país (tabela I).

A avaliação zoométrica, com o auxílio de hipômetro zootécnico e fita métrica, foi feita por meio de medidas cranianas e morfoestruturais em vacas adultas dos rebanhos, ou seja, com mudas dentárias completas (acima de cinco anos de idade), e desenvolvimento corporal completo. Foram também calculados índices corporais referentes às relações entre algumas medidas zoométricas.

Foram realizadas as seguintes medidas cranianas e morfoestruturais (Herrera, 2002):

- Comprimento de orelha $(\mathrm{CO})=$ distância entre a base de inserção cranial do pavilhão auricular e sua extremidade;

- Largura da orelha $(\mathrm{LO})=$ distância entre as bordas da largura maior do pavilhão auricular, com curvatura natural mantida;

- Base de chifres $(B C)=$ distância entre os pontos de inserção dos dois cornos no crânio;

- Arco de chifres $(\mathrm{AC} 2)=$ distância entre as duas extremidades livres dos cornos;

- Comprimento da cabeça $(C C 2)=$ distância entre osso occipital e o lábio superior;

- Largura da cabeça (LC)= distância máxima entre os arcos zigomáticos;

- Comprimento da cara (CC3)= distância entre linha traçada entre os arcos zigomáticos e o lábio superior;

- Altura de cernelha $(\mathrm{AC})=$ ponto mais alto da região interescapular, imediatamente caudal à giba ou cupim, até o solo;

- Altura de garupa (AG)= ponto mais alto da tuberosidade sacral do íleo até o solo;

- Comprimento corporal $(\mathrm{CC})=$ traçado entre a cabeça do úmero e a ponta do ísquio;

- Perímetro torácico $(\mathrm{PT})=$ circunferência do tórax passando pelo cilhadouro;

- Comprimento de garupa $(C G)=$ traçado entre a asa do íleo e a ponta do ísquio;

Os índices corporais foram obtidos pelas seguintes relações (Herrera, 2002):

- Índice corporal (IC)= razão entre as medidas CC e PT, expressa em percentagem;

- Índice de proporcionalidade (IP)= razão entre as medidas AC e CC, expressa em percentagem;

- Índice de conformação (IC2)= razão entre as medidas PT e AC;

- Peso estimado $(\mathrm{PE})=$ resultado do produto (perímetro torácico $)^{3}$ x 80;

- Índice de compacidade (IC3)= razão entre PE e AC, expressa em percentagem.

Os dados foram submetidos à análise de variância e teste de médias (Tukey $5 \%$ de probabilidade) para avaliar o efeito de rebanho com o auxílio do SAS (PROC GLM SAS, 2006). Posteriormente, foi feita análise multivariada de componentes principais, com formação de dendograma UPGMA, para verificar a formação de grupos de similaridade entre os mesmos, por meio do programa MVSP 3.1 (Manly, 2008). 
Tabela II. Médias de comprimento de orelha (CO), largura da orelha (LO), base de chifres (BC), arco de chifres (AC2), comprimento da cabeça (CC2), largura da cabeça (LC) e comprimento da cara (CC3), de fêmeas adultas da raça Sindi provenientes de cinco rebanhos de base de formação conhecida (Means for ear length (CO), ear width (LO), horns base (BC), horns arc (AC2), head length (CC2), head width (LC), and face length (CC3) of Sindi breed adult females from five known formation basis herds).

\begin{tabular}{|c|c|c|c|c|c|c|c|}
\hline \multirow{2}{*}{ Variáveis $(\mathrm{cm})$} & \multicolumn{5}{|c|}{ Rebanhos } & \multirow{2}{*}{ Média $\pm D P$} & \multirow{2}{*}{ CV $(\%)$} \\
\hline & 1 & 2 & 3 & 4 & 5 & & \\
\hline $\mathrm{CO}$ & $26,01^{d}$ & $26,31^{\mathrm{cd}}$ & $29,21^{a}$ & $28,70^{\mathrm{ab}}$ & $27,49^{\mathrm{bc}}$ & $27,40 \pm 2,35$ & 7,4 \\
\hline LO & $12,24^{a}$ & $12,80^{a}$ & $12,66^{a}$ & $12,76^{\mathrm{a}}$ & $12,33^{a}$ & $12,50 \pm 1,07$ & 8,4 \\
\hline $\mathrm{BC}$ & $16,91^{\mathrm{b}}$ & $17,05^{b}$ & $17,81^{\mathrm{b}}$ & $21,14^{\mathrm{a}}$ & $17,58^{\mathrm{b}}$ & $17,90 \pm 2,54$ & 12,0 \\
\hline $\mathrm{AC} 2$ & $27,58^{b}$ & $29,14^{\mathrm{ab}}$ & $30,53^{\mathrm{ab}}$ & $33,07^{a}$ & $29,70^{\mathrm{ab}}$ & $29,80 \pm 7,15$ & 23,6 \\
\hline $\mathrm{CC} 2$ & $43,95^{d}$ & $44,85^{d}$ & $53,61^{a}$ & $47,44^{c}$ & $50,96^{\mathrm{b}}$ & $48,10 \pm 4,24$ & 4,4 \\
\hline LC & $20,41^{b}$ & $19,51^{\mathrm{cd}}$ & $19,98^{\mathrm{bc}}$ & $22,09^{a}$ & $19,25^{\mathrm{d}}$ & $20,10 \pm 1,42$ & 5,4 \\
\hline $\mathrm{CC} 3$ & $38,24^{b}$ & $37,30^{\mathrm{b}}$ & $40,84^{a}$ & $39,83^{a}$ & $39,66^{a}$ & $39,10 \pm 2,40$ & 5,3 \\
\hline
\end{tabular}

1= EMEPA-Alagoinha, PB; 2 = CPATSA-Petrolina, PE; $3=$ Fazenda Lagoa Formosa-Novo Horizonte, SP; $4=$ Fazenda NUPEÁRIDO-Patos, PB; $5=$ Fazendinha-Mogeiro, PB. abMédias seguidas de letras diferentes na mesma linha diferem estatisticamente $(p<0,05)$ pelo teste de Tukey.

\section{RESULTADOS E DISCUSSÃO}

Na tabela II estão apresentadas as médias por rebanho, média geral, desvio padrão e coeficientes de variação $(\mathrm{CV})$ das medidas cranianas de fêmeas adultas da raça Sindi. Embora não tenha nenhum elemento que sirva de indicativo produtivo do animal, é na cabeça onde se encontra o maior número de regiões que apresentam variação em relação a tamanho, conformação, posição e inserção, sendo suficientes para a caracterização racial do indivíduo (Peixoto et al., 1990). Não se encontram trabalhos de caracterização racial de rebanhos Sindi no Brasil ou em outras partes do mundo disponíveis na literatura, de forma que as discussões serão feitas utilizando-se como base as inferências feitas à essas características no padrão oficial da raça no país, homologado pela Associação Brasileira de Criadores de Zebu (ABCZ), embora este não estabeleça valores numéricos exatos para as medidas tomadas.

As variáveis cranianas estudadas apresentaram um baixo coeficiente de variação, o que indica uma boa homogeneidade dos dados nas populações estudadas. É possível notar, contudo, a existência de uma diferenciação dessas medidas entre as populações em questão, já que apenas para a variável de largura de orelha (LO) não houve diferença entre os rebanhos $(p>0,05)$.

Os rebanhos 3 e 4 apresentaram médias de medidas cranianas maiores do que aqueles de descendência direta dos animais trazidos na importação oficial do IAN em 1952 (rebanhos 1 e 2). O rebanho da fazenda Fazendinha, formado por cruzamentos dessas duas bases da raça no Brasil, apresentou médias intermediárias, podendo tender para uma proximidade ou igualdade com qualquer uma delas de acordo com a variável em análise.

Segundo o padrão racial homologado na $\mathrm{ABCZ}$, as orelhas dos bovinos da raça Sindi devem apresentar tamanho médio, serem largas e um pouco pendentes, descrição na qual se encaixam todas as medidas encontradas, observando-se uma média geral para o comprimento de $27,4 \mathrm{~cm}( \pm 2,35)$ e $12,5 \mathrm{~cm}( \pm 1,07)$ para a largura (Leite et al., 2001; Leite, 2006).
A medida de base de chifres (BC) está diretamente relacionada com o tamanho e desenvolvimento do que se conhece zootecnicamente como região da marrafa do bovino e apresentou média geral de $17,9 \mathrm{~cm}( \pm 2,54)$, existindo diferença estatística apenas entre o rebanho 4 e os demais, cujo valor médio obtido em seus animais foi de $21,14 \mathrm{~cm}$.

Já na medida AC2 (arco de chifres), que se refere à distância entre as extremidades dos cornos, a média geral foi de $29,8 \mathrm{~cm}( \pm 7,15)$, sem existir um padrão de desenvolvimento entre as populações de acordo com sua base de formação. Essa característica é realmente bastante variável, sendo fortemente influenciada pela idade do animal, tendendo a diminuir cada vez mais nas fêmeas à medida que os animais vão envelhecendo, pois o crescimento dos cornos tende a aproximar suas extremidades.

Em relação às proporções gerais da cabeça de fêmeas Sindi, está registrado que esta deve ser curta, de tamanho médio e bem proporcional, com fronte de largura média, chanfro reto e perfil sub convexo, notando-se uma adequação dos animais avaliados pelas médias gerais de comprimento e largura da cabeça e comprimento da cara.

O comprimento da cabeça (CC2) apresentou média geral de $48,1 \mathrm{~cm}( \pm 4,24)$, com diferença significativa $(p<0,05)$ entre os rebanhos, sendo maior naqueles formados a partir do núcleo de seleção da família Castilho ( 3 e 4) e no de formação mista (5) e menor nos rebanhos formados a partir de descendentes de animais importados em 1952 ( 1 e 2). Com média geral de $39,1 \mathrm{~cm}( \pm 2,40)$, a medida CC3 (comprimento da cara) apresentou um padrão de resposta semelhante ao da medida CC2.

A largura do crânio do animal (LC) apresentou valor médio geral de $20,1 \mathrm{~cm}( \pm 1,42)$. A LC foi maior no rebanho 4 e 1 . Essas, por sua vez, foram maiores que a apresentada no rebanho 3. Prevalecendo médias maiores que os provenientes da importação do IAN (2). Nesta medida, a menor média foi registrada no rebanho de origem mista (5). 
Tabela III. Médias de altura de cernelha (AC), altura de garupa (AG), comprimento corporal (CC), perímetro torácico (PT) e comprimento de garupa (CG) de fêmeas adultas da raça Sindi provenientes de cinco rebanhos de base genética de formação conhecida (Means for withers height (AC), croup height (AG), body lenght (CC), girth (PT) and croup lenght (CG) of Sindi breed adult females from five known formation basis herds).

\begin{tabular}{|c|c|c|c|c|c|c|c|}
\hline \multirow{2}{*}{ Variáveis (cm) } & \multicolumn{5}{|c|}{ Rebanhos } & \multirow{2}{*}{ Média $\pm D P$} & \multirow{2}{*}{ CV $(\%)$} \\
\hline & 1 & 2 & 3 & 4 & 5 & & \\
\hline$A C$ & $120,49^{d}$ & $117,70^{c}$ & $132,41^{a}$ & $129,71^{\mathrm{b}}$ & $127,80^{b}$ & $125,00 \pm 6,58$ & 2,7 \\
\hline$A G$ & $122,99^{d}$ & $120,15 \mathrm{e}$ & $137,04^{\mathrm{a}}$ & $132,09^{b}$ & $129,47^{c}$ & $127,70 \pm 6,98$ & 2,6 \\
\hline $\mathrm{CC}$ & $132,42^{b}$ & $131,77^{b}$ & $146,79^{a}$ & $144,87^{a}$ & $136,11^{b}$ & $137,50 \pm 13,79$ & 9,1 \\
\hline PT & $159,41^{c}$ & $151,84^{d}$ & $182,08^{a}$ & $184,47^{a}$ & $166,59^{b}$ & $167,00 \pm 15,05$ & 5,2 \\
\hline CG & $42,38^{c}$ & $41,36^{c}$ & $50,38^{a}$ & $44,44^{\mathrm{b}}$ & $44,00^{\mathrm{b}}$ & $44,30 \pm 3,97$ & 5,7 \\
\hline
\end{tabular}

1= EMEPA-Alagoinha, PB; 2 = CPATSA-Petrolina, PE; 3 = Fazenda Lagoa Formosa-Novo Horizonte, SP; 4 = Fazenda NUPEÁRIDO-Patos, PB; $5=$ Fazendinha-Mogeiro, PB. abMédias seguidas de letras diferentes na mesma linha diferem estatisticamente $(p<0,05)$ pelo teste de Tukey.

Tabela IV. Médias de índice corporal (IC), índice de proporcionalidade (IP), índice de conformação (IC2), peso estimado (PE) e índice de compacidade (IC3) de fêmeas adultas da raça Sindi provenientes de cinco rebanhos de base genética de formação conhecida (Means for body mass index (IC), proportionality index (IP), conformation index (IC2), estimated weight (PE) and compacity index (IC3) of Sindi breed adult females from five known formation basis herds).

\begin{tabular}{|c|c|c|c|c|c|c|c|}
\hline \multirow{2}{*}{ Variáveis $(\mathrm{cm})$} & \multicolumn{5}{|c|}{ Rebanhos } & \multirow{2}{*}{ Média $\pm D P$} & \multirow{2}{*}{ CV $(\%)$} \\
\hline & 1 & 2 & 3 & 4 & 5 & & \\
\hline IC & $83,11^{a b}$ & $86,91^{a}$ & $79,81^{b}$ & $79,74^{b}$ & $81,84^{b}$ & $82,60 \pm 8,22$ & 9,5 \\
\hline IP & $91,06^{a}$ & $89,44^{\mathrm{a}}$ & $91,63^{\mathrm{a}}$ & $88,45^{a}$ & $107,01^{a}$ & $94,10 \pm 39,20$ & 41,3 \\
\hline IC2 & $1,32^{\mathrm{c}}$ & $1,29^{c}$ & $1,38^{b}$ & $1,42^{\mathrm{a}}$ & $1,30^{c}$ & $1,30 \pm 0,07$ & 4,5 \\
\hline PE & $324,97^{c}$ & $281,81^{d}$ & $489,54^{a}$ & $507,19^{a}$ & $372,19^{b}$ & $381,80 \pm 107,13$ & 16,8 \\
\hline IC3 & $269,41^{b}$ & $239,23^{c}$ & $369,35^{a}$ & $390,44^{a}$ & $290,69^{b}$ & $302,70 \pm 72,42$ & 15,3 \\
\hline
\end{tabular}

1= EMEPA-Alagoinha, PB; 2 = CPATSA-Petrolina, PE; $3=$ Fazenda Lagoa Formosa-Novo Horizonte, SP; $4=$ Fazenda NUPEÁRIDO-Patos, PB; $5=$ Fazendinha-Mogeiro, PB. abMédias seguidas de letras diferentes na mesma linha diferem estatisticamente $(p<0,05)$ pelo teste de Tukey.

As médias por rebanho, média geral, desvio padrão e coeficientes de variação (CV) das medidas morfoestruturais de fêmeas adultas da raça Sindi são apresentadas na tabela III. Diferente do estudo da morfologia, que se baseia na análise das formas do animal, a avaliação morfoestrutural analisa quantitativamente características exteriores do indivíduo, mostrando-se assim como uma ciência métrica objetiva (Herrera, 2002; Pacheco et al., 2008).

Foi observada uma diferença de, aproximadamente, três centímetros entre as duas alturas corporais consideradas, AC e AG, com médias gerais de $125,00 \mathrm{~cm}$ $( \pm 6,52)$ e $127,70 \mathrm{~cm}( \pm 6,98)$, respectivamente. Acompanhando a tendência das medidas cranianas, os rebanhos 3 e 4 apresentaram médias das medidas corporais superiores ao rebanho 5 (misto) e este, por sua vez, média superior aos rebanhos 1 e 2 .

O padrão de resposta foi semelhante para as demais variáveis morfoestruturais demonstrando, claramente, um maior desenvolvimento corporal dos animais oriundos da seleção da família Castilho em relação aos demais.

O comprimento corporal e o perímetro torácico são medidas denotativas de eficiência produtiva pois, vacas mais curtas e com maior perímetro torácico exigem menos alimento para mantença e tem ampla cavidade corporal para albergar sistema digestivo e cardiorrespiratório (Oliveira et al., 2003; Rocha et al., 2003). Comparando-se as medidas corporais obtidas com os dados de vacas Jersey (De Vuono, 2000), com comprimento corporal e perímetro torácico de $117 \mathrm{~cm}$ $( \pm 6,18)$ e $134 \mathrm{~cm}( \pm 7,2)$ respectivamente, torna-se patente a similaridade com uma raça bem conceituada do ponto de vista produtivo e que tem uma considerável distribuição nos continentes. A comparação tanto pode ser feita com genótipos locais, como é o caso da raça Curraleiro, que apresentou AC de 113,94 cm, CC de $130,50 \mathrm{~cm}$ e PT 166,94 cm (Abreu et al., 2005; Bianchini et al., 2006), quanto com vacas cruzadas vacas $5 / 8$ Holandês $+3 / 8 \mathrm{Gir}$, que apresentaram médias de AC, CC e PT $126,55( \pm 9,54), 129,13( \pm 15,99)$ e de $170,74( \pm 13,33)$ $\mathrm{cm}$, respectivamente (Barreto et al., 2004).

Os índices corporais de fêmeas adultas da raça Sindi são apresentados na tabela IV. Novamente, a falta de trabalhos de caracterização racial com bovinos, dessa e de outras raças, que contemplem o cálculo desses índices, é um fator que dificulta a comparação dos resultados.

O único índice para o qual os rebanhos não diferiram $(p>0,05)$ foi o de proporcionalidade (IP), com média geral de $94,10 \mathrm{~cm}( \pm 39,20)$. O rebanho 2 apresentou índice corporal (IC) médio superior a todos os rebanhos. Baseado na classificação feita por Herrera (2002), apenas o rebanho 2 poderia ser considerado mediolíneo, por apresentar IC médio entre 86 e 88, sendo todos os demais classificados como brevilíneos, por terem média de IC inferior a 85. 
Tabela V. Autovetores dos componentes principais 1 (CP1), 2 (CP2) e 3 (CP3) das medidas zoométricas de vacas da raça Sindi provenientes de cinco rebanhos de base genética de formação conhecida (Eigenvectors of principal components 1 (CP1), 2 (CP2), and 3 (CP3), for zoometric measures of Sindi cows from five herds of known genetic basis of formation).

\begin{tabular}{lccc}
\hline Variáveis & CP1 & CP2 & CP3 \\
\hline AC & 0,3198 & $-0,0369$ & $-0,2458$ \\
AG & 0,3222 & $-0,0144$ & $-0,2601$ \\
CC & 0,1855 & 0,5159 & $-0,0396$ \\
PT & 0,3522 & $-0,0540$ & 0,1109 \\
CG & 0,3015 & 0,0113 & $-0,1866$ \\
CO & 0,2149 & 0,0479 & $-0,1763$ \\
LO & 0,0598 & 0,0779 & 0,0134 \\
BC & 0,1594 & 0,0967 & 0,3327 \\
AC2 & 0,0775 & 0,1891 & 0,1931 \\
CC2 & 0,2601 & $-0,0280$ & $-0,4152$ \\
LC & 0,1586 & 0,0769 & 0,4537 \\
CC3 & 0,2191 & 0,0011 & $-0,1887$ \\
IC & $-0,1280$ & 0,5656 & $-0,1319$ \\
IP & $-0,0092$ & $-0,5845$ & $-0,0338$ \\
IC2 & 0,2618 & $-0,0543$ & 0,3940 \\
PE & 0,3505 & $-0,0462$ & 0,1312 \\
IC3 & 0,3387 & $-0,0485$ & 0,1980 \\
I & 7,46 & 2,58 & 1,69 \\
VA (\%) & 43,9 & 59,11 & 69,05 \\
\hline
\end{tabular}

I= Autovalor da matriz de correlação; VA= Variância acumulada.

Os rebanhos 3 e 4 mantêm a tendência já observada de maiores medidas, agora refletida no peso estimado e no índice de compacidade. Com relação ao índice de conformação (IC2), o rebanho 4 foi superior aos demais.

A média geral do peso estimado foi próxima dos $400 \mathrm{~kg}$, superior aos resultados relatados por De Vuono et al. (2000) para vacas da raça Jersey, considerada uma raça de pequeno porte como a Sindi, que foi de 298,65 kg. Os rebanhos com origem na seleção da família Castilho concorreram expressivamente para elevar essa média. As diferenças entre os rebanhos, em função de suas distintas bases, pode ser resultado de procedimentos diferenciados quanto ao rigor aplicado na preservação do padrão racial associado ao porte, principalmente.

As fêmeas dos rebanhos 3 e 4 podem, inclusive, ser comparadas em porte com vacas $5 / 8$ Holandês + $3 / 8$ Gir que apresentaram peso médio de $326,52 \mathrm{~kg}$ (Barreto et al., 2004); ou com vacas da raça Guzerá, que apresentram média de 399,61 kg de peso vivo (Okuda, 1992); ou até mesmo da raça Nelore que no, estudo de Freneau et al. (2008), apresentaram peso médio de $434,88 \mathrm{~kg}( \pm 56,65)$.

Na tabela V são apresentados os autovetores da análise de componentes principais dos dados zoométricos. Utilizando estes autovetores foi construída a figura 1, que agrupa os rebanhos de acordo com a distância euclidiana, em relação à similaridade e interrelação entre as medidas.

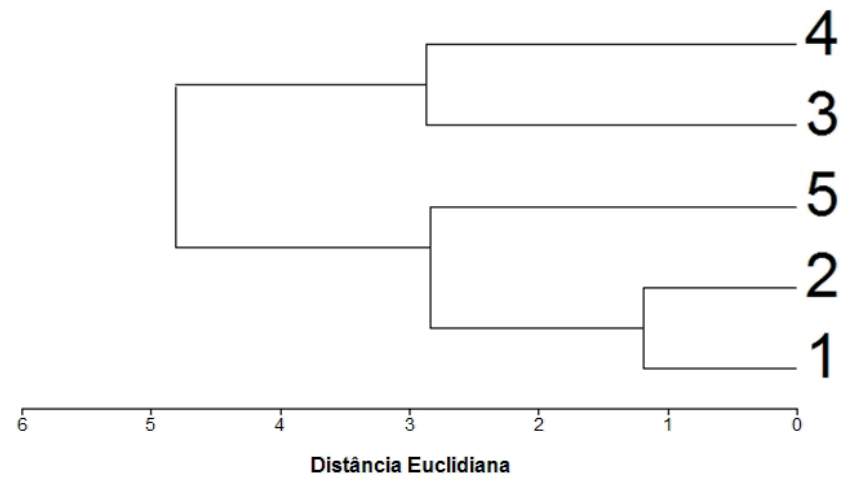

Figura 1. Formação de agrupamentos de cinco rebanhos da raça Sindi, de acordo com as medidas zoométricas de suas vacas. Rebanhos: 1= EMEPA-Alagoinha, PB; 2 = CPATSA-Petrolina, PE; $3=$ Fazenda Lagoa Formosa-Novo Horizonte, SP; $4=$ Fazenda NUPEARIDO-Patos, PB; $5=$ Fazendinha-Mogeiro, PB (Groups formation of five Sindi breed herds according to the zoometric measures of their cows. Herds: 1= EMEPA-Alagoinha, PB; 2= CPATSA-Petrolina, PE; 3= Fazenda Lagoa Formosa-Novo Horizonte, SP; 4= Fazenda NUPEÁRIDOPatos, PB; 5= Fazendinha-Mogeiro, PB).

$\mathrm{Na}$ figura 1 observa-se o agrupamento dos rebanhos que são descendentes diretos dos animais importados pelo IAN no ano de 1952 (1 e 2) e dos rebanhos oriundos da seleção da raça Sindi no Brasil feito pela família Castilho (3 e 4), comprovando a fixação dos caracteres corporais pelo processo de seleção exercido sobre os mesmos. As diferentes tendências produtivas, exemplificada pela produção leiteira do rebanho 1 (Lemos et al., 2006) e pela produção de carne do rebanho 4 (Leite, 2007), somam-se a evidência da presença de duas linhagens desenvolvidas na raça Sindi no Brasil, uma com menores e outra com maiores proporções corporais. O rebanho da fazenda Fazendinha, tomado como referência de um criatório formado pelas duas linhas da raça no país, se comporta de forma intermediária, de forma que seus indivíduos mostram-se como um elemento de caracterização fenotípica média, por possuir influência de ambas.

Vale ressaltar que os resultados encontrados não podem ser creditados a diferenciação nas condições de criação entre os rebanhos, visto que estas são distintas mesmo para aqueles que foram agrupados como semelhantes. Os rebanhos 1 e 2 estão alocados, respectivamente, nas cidades de Alagoinha, na região Agreste do Estado da Paraíba com pluviosidade média acima de $1000 \mathrm{~mm}$ por ano, e de Petrolina, no Sertão do Estado de Pernambuco, com pluviosidade média abaixo de $500 \mathrm{~mm}$. Já os rebanhos 3 e 4 estão alocados, respectivamente, nas cidades de Novo Horizonte, no Oeste do Estado de São Paulo, com pluviosidade média acima de 1500 mm, e de Patos, no Sertão do Estado da Paraíba, com pluviosidade média anual abaixo de $600 \mathrm{~mm}$.

\section{CONCLUSÃO}

Os resultados evidenciam para a presença de duas linhagens desenvolvidas na raça no Brasil. A linhagem proveniente do Instituto Agronômico do Norte, com cabeça e crânio mais leve e menores proporções corporais e a linhagem proveniente da família Cas- 
tilho, compreendendo animais maiores e com cabeça e crânios mais volumosos. A diferença entre as duas linhagens foram, sem dúvida, reflexo dos processos seletivos aplicados aos dois grupos.

\section{AGRADECIMENTOS}

Pesquisa Financiada pelo BNB/FUNDECI/ETENE. $\mathrm{CNPq}$

Graças a bolsas de Produtividade em Pesquisa/

Ao BNB/FUNDECI/ETENE, a Associação Brasileira de Criadores de Sindi (ABCSINDI) e aos responsáveis pelos rebanhos estudados, e a todos que colaboraram para a realização da pesquisa.

\section{BIBLIOGRAFIA}

Abreu, U.G.P.; Santos, S.A.; Sereno, J.R.B.; Comastri-Filho, J.A. e Ravanelli, M.S. 2005. Caracterización morfométrica de los bovinos Pantaneiros del núcleo de conservación in situ de Nhumirim. Arch Zootec, 54: 211-216.

Barreto, M.B.P.; Santos, R.S.B.; Wischral, A. e Cavalcanti Júnior, J.A. 2004. Pelvimetria e pelvilogia em fêmeas bovinas da raça girolanda em diferentes estágios reprodutivos. Cie Vet Nos Trop, 7: 131-139.

Bianchini, E.; McManus, C.; Lucci, C.M.; Fernandes, M.C.B.; Prescott, E.; Mariante, A.S. e Egito, A.A. 2006. Características corporais associadas com a adaptação ao calor em bovinos naturalizados brasileiros. Pesq Agropec Bras, 41: 1443-1448.

Bodó, I. 1990. Methods and experiences with in situ preservation of farm animals. Expert consultation on FAO programmes for the preservation of animal genetic resources.

De Vuono, R.S. 2000. Pelvimetria e pelviologia em vacas Jersey. Dissertação (Mestrado). Faculdade de Medicina Veterinária e Zootecnia. Universidade de São Paulo. São Paulo. 56 pp.

Freneau, G.E.; Silva, J.C.C.; Borjas, A.L.R. e Amorim, C. 2008. Estudo de medidas corporais, peso vivo e condição corporal de fêmeas da raça nelore Bos taurus indicus ao longo de doze meses. Cie Anim Bras, 9: 76-85

Herrera, M. 2002. Criterios etnozootécnicos para la definición de poblaciones animales. In: V Congreso Ibérico Sobre Recursos Genéticos Animales. 2002. Anais... INIA. Madrid. pp. 41-48.
Leite, P.R. de M.; Santiago, A.A.; Navarro Filho, H.R.; Albuquerque, R.P. de F. e Leite, R. de M.H. 2001. Sindi: gado vermelho para o semiárido. Ed. EMEPA-PB. Banco do Nordeste. João Pessoa-PB. 147 pp. Leite, P.R.M. 2006. Mudanças no padrão racial do Sindi. Rev Agropec Tropic, 147: 41

Leite, P.R.M. 2007. Sindi dá show no frigorífico. Rev Agropec Tropic, 152: $73-74$

Lemos, P.F.B.; Leite, S.V.F.; Albuquerque, R.P.F.; Guedes, P.L.C.; Lima, A.M.; Fernandes, F.G. e Guedes Filho, E.A. 2007. O Zebu leiteiro da EMEPA-PB. Características produtivas, reprodutivas e sistema de produção. João Pessoa-PB. 20 pp.

Manly, B.J.F. 2008. Métodos estatísticos multivariados: uma introdução. 3. ed. Ed. Artmed. Porto Alegre-RS. 230 pp.

Okuda, H.T. 1992. Aspectos de pelvimetria e pelviologia em fêmeas de bovinos da raça Guzerá (Bos indicus Linnaeus, 1758). Tese (Doutorado). Faculdade de Medicina Veterinária e Zootecnia. Universidade de São Paulo. São Paulo. 45 pp.

Oliveira, P.C.; Bombonato, P.P. e Balieiro, J.C.C. 2003. Pelvimetria em vacas Nelore. Braz J Vet Res Anim Sci, 40: 297-304.

Pacheco, A.; Quirino, C.R.; Pinheiro, O.L.V.M. e Almeida, J.V.C. 2008. Medidas morfométricas de touros jovens e adultos da raça Guzerá. Rev Bras Saúde Prod Anim, 9: 426-435.

Peixoto, A.M.; Lima, F.P.; Tosi, H. e Sampaio, N.S. 1990. Exterior e julgamento de bovinos. FEALQ. Piracicaba. 222 pp.

Ribeiro, M.N.; Gomes Filho, M.A.; Bermejo, J.V.D.; Vallejo, M.E.C.; Oliveira, J.C.V.; Cruz, G.R.B.; Rocha, L.L. da; Silva, R.C.B. da; Menezes, M.P.C.; Ferreira, M.P. de B. e Pimenta Filho, E.C. 2004. Conservação de raças caprinas no Brasil: histórico, situação atual e perspectivas. UFPE. Imprensa Universitária. Recife/PE. 62 pp.

Rocha, E.D.; Andrade, V.J.; Euclides Filho, K.; Nogueira, E. e Figueredo, G.R. 2003. Mature Nellore cow size its effect on beef cattle production system. Arq Bras Med Vet Zootec, 55: 474-479.

Statistical Analysis System - SAS. 2008. SAS system for Windows. Version 9.2. SAS Institute (CD-ROM). Cary, NC.

Torres, A.D.P. e Jardim, W.R. 1982. Manual de zootecnia: Raças que interessam ao Brasil, bovinas, zebuínas, bubalinas, cavalares, asininas, suínas, ovinas, caprinas, cunícolas, avícolas. 2.ed. Ed. Agronômica Ceres. Campinas/SP. 303 pp. 